OPEN ACCESS

Edited by:

Jianzhong Su,

Wenzhou Medical University, China

Reviewed by:

Jie Zhang,

Tongii University, China

Hao Lin,

University of Electronic Science and Technology of China, China

*Correspondence:

Yina Zhang

yinazhlu@163.com

${ }^{\dagger}$ These authors share first authorship

Specialty section: This article was submitted to Epigenomics and Epigenetics, a section of the journal Frontiers in Cell and Developmental Biology

Received: 03 February 2020 Accepted: 04 March 2020

Published: 31 March 2020

Citation:

Zhang $M$, Cheng $L$ and Zhang $Y$ (2020) Characterization of Dysregulated IncRNA-Associated ceRNA Network Reveals Novel IncRNAs With ceRNA Activity as Epigenetic Diagnostic Biomarkers for Osteoporosis Risk.

Front. Cell Dev. Biol. 8:184. doi: 10.3389/fcell.2020.00184

\section{Characterization of Dysregulated} IncRNA-Associated ceRNA Network Reveals Novel IncRNAs With ceRNA Activity as Epigenetic Diagnostic Biomarkers for Osteoporosis Risk

\author{
Meijie Zhangt, Luyang Cheng ${ }^{\dagger}$ and Yina Zhang* \\ Department of Geriatrics, The Second Affiliated Hospital of Harbin Medical University, Harbin Medical University, Harbin, \\ China
}

The altered expression of long non-coding RNAs (IncRNAs) has been implicated in the development and human diseases. However, functional roles and regulatory mechanisms of IncRNA as competing endogenous RNAs (ceRNAs) in osteoporosis and their potential clinical implication for osteoporosis risk are largely unexplored. In this study, we performed integrated analysis for paired expression profiles and regulatory relationships of dysregulated IncRNAs, mRNAs, and miRNAs based on "ceRNA hypothesis," and constructed an osteoporosis-related dysregulated miRNA-mediated IncRNA-mRNA ceRNA network (DysCeNet) composed of 105 nodes (including eight miRNAs, 24 mRNAs, and 73 IncRNAs) and 515 edges. Functional analysis suggested that the DysCeNet was involved in known osteoporosis or bone metabolism-related biological processes and pathways. Then, we performed random forest-based feature selection for 73 IncRNAs with ceRNA activity and identified 25 of 73 IncRNAs as potential diagnostic biomarkers. A random forest-based classifier composed of 25 IncRNA biomarkers (RF-25IncRNA) was developed for predicting osteoporosis risk. Performance evaluation with the leave-one-out cross-validation (LOOCV) procedure showed that the RF-25IncRNA achieved a good performance in distinguishing highand low-bone mineral density (BMD) subjects in different osteoporosis datasets. Our study for the first time revealed a global view of IncRNA-associated ceRNA regulation in osteoporosis and provided novel IncRNAs with ceRNA activity as candidate epigenetic diagnostic biomarkers for early detection of osteoporosis risk.

Keywords: competing endogenous RNAs, long non-coding RNAs, osteoporosis, biomark, epigenetics

\section{INTRODUCTION}

Osteoporosis is a progressive systemic skeletal disease with low bone density and deterioration of bone architecture (Garnero, 2017; Tu et al., 2018). It is estimated that over 14 million men and women in the United States will have osteoporosis by 2020 (Burge et al., 2007). People with osteoporosis substantially have an increased risk of bone fragility and fracture, leading to increase 
pain, disability, nursing home placement, total health care costs, and mortality (Tu et al., 2018). Early detection and intervention for osteoporosis risk are an effective way to delay the development of disease and improve the quality of life of patients. Therefore, there is an urgent need to identify novel molecular biomarkers in the clinical assessment of osteoporosis risk.

With the development and application of high-throughput omics technologies, it has been shown that osteoporosis has genetic and molecular heterogeneity like many other common complex diseases, and disruption in some molecular pathways can disturb the equilibrium of bone turnover and thereby contribute to osteoporosis (Al Anouti et al., 2019). Long non-coding RNAs (lncRNAs) are a newly discovered class of non-coding RNAs (ncRNAs) that are longer than $200 \mathrm{bp}$ (Pelechano and Steinmetz, 2013) and have attracted much attention in recent medical studies. A large number of studies have demonstrated that lncRNAs is an important player of the genomic regulatory network and is involved in a wide variety of biological progress (Mercer and Mattick, 2013; Guo et al., 2016; Quinn and Chang, 2016; Bunch, 2018; Sun et al., 2020). IncRNAs have been attributed to various functions in development, differentiation, and human disease by negatively or positively regulating gene expression at the transcriptional, post-transcriptional, and epigenetic levels (Kornienko et al., 2013; Maass et al., 2014). Growing functional roles of lncRNAs has been highlighted in osteoporosis during recent studies. For example, XIST, a well-known major effector of the X-inactivation process, was recently reported to promote osteoporosis through inhibiting bone marrow mesenchymal stem cell differentiation (Chen et al., 2019). Mei et al. (2019) found that lncRNA ZBTB40-IT1 played a critical role in bone metabolism and can be modulated by osteoporosis GWAS risk SNPs suppresses osteogenesis. It has become increasingly clear that lncRNAs can act as competing endogenous RNAs (ceRNAs) to interact other RNA molecules by competing for binding to shared microRNAs, which has been implicated in development and human disease including osteogenesis (Tay et al., 2014; Huang et al., 2019a,b; Silva et al., 2019). However, genome-wide exploration for miRNA-mediated lncRNA-associated ceRNA mechanism in osteoporosis and their potential clinical implication for osteoporosis risk remained largely unknown.

In this study, we tried to construct a global miRNAmediated lncRNA-mRNA ceRNA network in the development of osteoporosis by integrating paired expression profiles and regulatory relationship of lncRNAs, mRNAs, and miRNAs based on "ceRNA hypothesis," and further to uncover novel lncRNAs with ceRNA activity as epigenetic diagnostic biomarkers for identifying people at high risk for developing osteoporosis.

\section{MATERIALS AND METHODS}

\section{Patients and Samples}

Ten samples [including five high-bone mineral density (BMD) subjects and five low-BMD subjects] with corresponding transcriptome gene expression microarray data (Affymetrix Human Exon 1.0 ST Array) and epigenomic miRNA microarray data (Affymetrix Multispecies miRNA-2 Array) were obtained from the Gene Expression Omnibus (GEO) database (the accession number is GSE62589 ${ }^{1}$ ). Two other osteoporosis datasets with transcriptome gene expression microarray data were obtained from the GEO database, including GSE56814 dataset (16 high-BMD subjects and 15 low-BMD subjects) (the accession number is GSE56814 ${ }^{2}$ ) and GSE13850 dataset (10 highBMD subjects and 10 low-BMD subjects) (the accession number is GSE $13850^{3}$ ).

\section{Acquisition and Analysis of Expression Profiles}

Raw transcriptome gene expression microarray data (CEL files) profiled on Affymetrix GeneChip Human Exon 1.0 ST Array (HuEx-1_0-st) and Affymetrix Human Genome U133A Array (HG-U133A) were obtained from the GSE63402 and GSE13850. These raw data were processed and normalized using the Robust Multichip Average (RMA) algorithm of $\mathrm{R}$ package "oligo" for background subtraction, quantile normalization, and summarization. Then, all probes of microarray were mapped into protein-coding genes using the $\mathrm{R}$ package "biomaRt" (Durinck et al., 2009). LncRNA expression profiles were obtained using repurposing strategy by mapping array probes into the human genome (GRCh 38) and lncRNA annotations from the GENCODE database ${ }^{4}$ (Zhou et al., 2016) using SeqMap tool (Jiang and Wong, 2008). Human mature miRNAs were retrieved from the miRNA microarray. Finally, expression profiles of 20,068 mRNA, 7821 lncRNAs, and 1100 miRNA were obtained for further analysis.

Differential expression analysis of lncRNAs, miRNAs, and mRNAs between high- and low-BMD subjects was performed using the R package "limma" (Ritchie et al., 2015). Those lncRNAs, miRNAs, and mRNAs with $p<0.05$ were considered as differentially expressed genes. Hierarchical clustering was performed to investigate the expression patterns between highand low-BMD subjects.

\section{Construction of Dysregulated IncRNA-Associated ceRNA Network}

The experimentally validated miRNA-mRNA and miRNAlncRNA interaction data were collected from the TarBase database $^{5}$ (Li et al., 2014). The dysregulated lncRNA-associated ceRNA network (DysCeNet) in osteoporosis was constructed based on the "ceRNA hypothesis" as follows: (i) Pearson correlation coefficient (PCC) was calculated to measure the expression correlation between differentially expressed mRNAs and lncRNAs from matched mRNA and lncRNA expression profiles. Those dysregulated lncRNA-mRNA pairs with PCC $>0.5$ were considered as candidate co-dysregulated lncRNA-mRNA ceRNA crosstalk; (ii) expression correlation between differentially expressed miRNAs and differentially

\footnotetext{
${ }^{1}$ https://www.ncbi.nlm.nih.gov/geo/query/acc.cgi?acc=GSE62589

${ }^{2}$ https://www.ncbi.nlm.nih.gov/geo/query/acc.cgi?acc=GSE56814

${ }^{3}$ https://www.ncbi.nlm.nih.gov/geo/query/acc.cgi?acc=GSE13850

${ }^{4}$ https://www.gencodegenes.org/

${ }^{5}$ http://starbase.sysu.edu.cn/
} 
expressed mRNAs, and between differentially expressed miRNAs and differentially expressed lncRNAs was evaluated using PCC; (iii) for a candidate co-dysregulated lncRNA-mRNA ceRNA crosstalk, both mRNAs and lncRNAs in this lncRNA-mRNA ceRNA crosstalk are targeted and co-expressed negatively with a common miRNA; this candidate co-dysregulated lncRNA-mRNA ceRNA crosstalk was selected as dysregulated miRNA-mediated lncRNA-mRNA ceRNA crosstalk; (iv) all miRNA-mediated lncRNA-mRNA ceRNA crosstalks were integrated to form a global DysCeNet.

\section{Identification of IncRNA Biomarkers With ceRNA Activity Using a Machine Learning Method}

To identify potential lncRNA biomarkers with ceRNA activity, a random forest approach and leave-one-out cross-validation (LOOCV) were used to select optimal lncRNAs biomarkers using the $\mathrm{R}$ package "randomForest" and out-of-bag (OOB) error, which measure the performance of the model on the training set (Lv et al., 2019; Tan et al., 2019). The OOB error will produce an unbiased estimate for the classification error, while the bagging method will decrease the chance of overfitting (Toth et al., 2019). Then, a random forest-based classifier was built using the optimal lncRNA biomarkers, and a receiver operating characteristic (ROC) curve and the area under ROC curve (AUC) was used to measure the diagnostic performance of the lncRNA classifier (Lai et al., 2019).

\section{Functional Enrichment Analysis}

Functional enrichment analysis of Gene Ontology (GO) and Kyoto Encyclopedia of Genes and Genomes (KEGG) for mRNAs in the DysCeNet was conducted to predict potential biological functions of lncRNAs in the DysCeNet using the $\mathrm{R}$ package "clusterprofiler" (Yu et al., 2012). Those significantly enriched GO terms with $p<0.05$ with mutually overlapping gene sets were clustered together using the Enrichment Map plugin in Cytoscape environment (Merico et al., 2010).

\section{RESULTS}

\section{Identification of Differentially Expressed mRNAs, miRNAs, and IncRNAs Associated With Osteoporosis}

To identify potential risk mRNAs, miRNAs, and lncRNAs in osteoporosis, we performed a comparative analysis for expression profiles of mRNAs, miRNAs, and lncRNAs between highand low-BMD subjects. A total of 68 mRNAs, 11 miRNAs, and 95 lncRNAs were identified as differentially expressed $(p<0.05)$ in high-BMD subjects compared with low-BMD

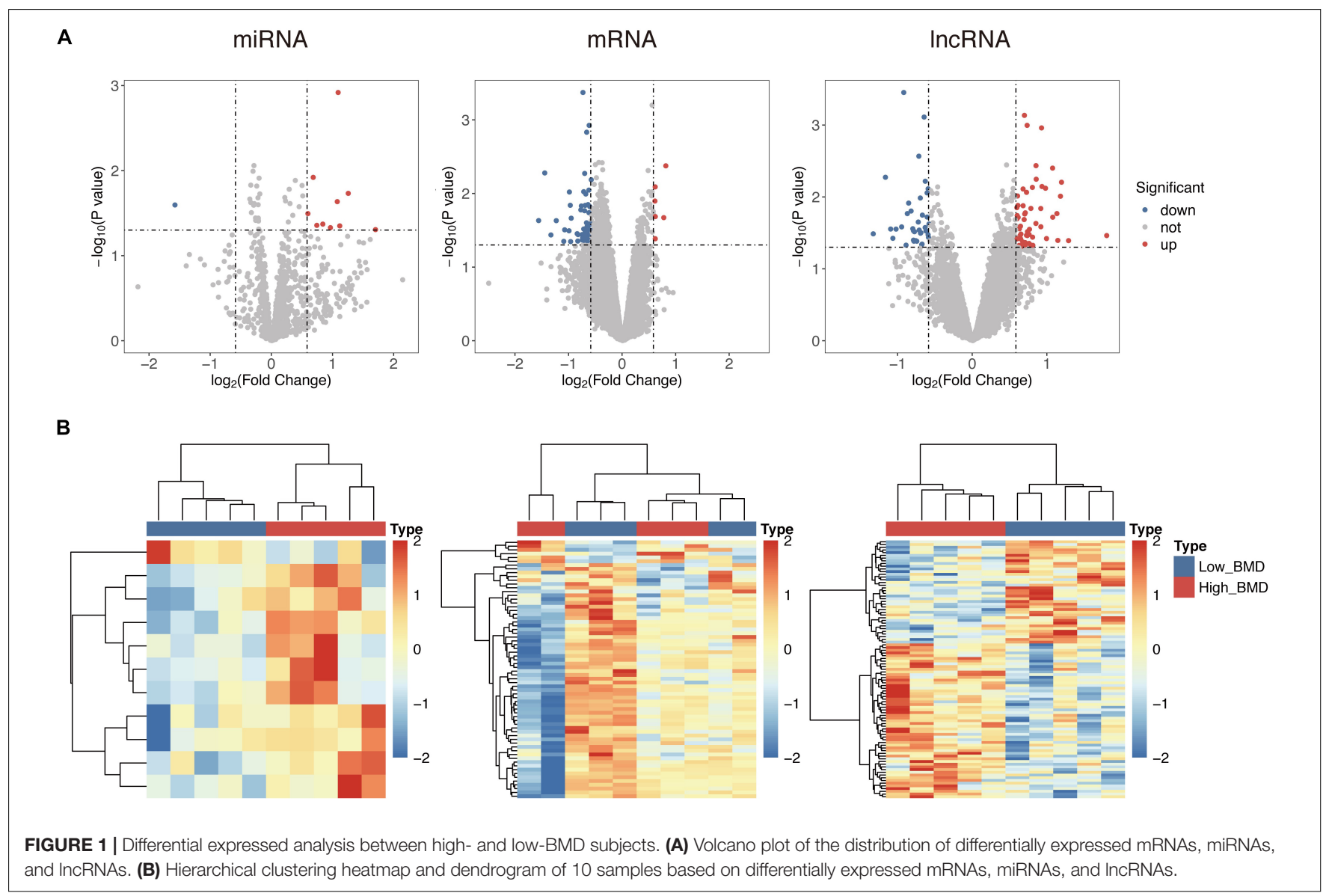


subjects (Supplementary Table S1). Among them, there were 73 unregulated genes (including six mRNAs, 10 miRNAs, and 57 lncRNAs) and 101 downregulated genes (including 62 mRNAs, one miRNA, and 38 lncRNAs) in high-BMD subjects compared with low-BMD subjects (Figure 1A). Hierarchical clustering analysis showed that expression patterns of these differentially expressed mRNAs, miRNAs, and lncRNAs were capable of distinguishing high-BMD subjects from low-BMD subjects (Figure 1B).

\section{Construction and Analysis of Dysregulated IncRNA-Associated ceRNA Network}

To construct an osteoporosis-related dysregulated miRNAmediated lncRNA-mRNA ceRNA network, we performed an integrated analysis for paired expression profiles and regulatory relationships of dysregulated lncRNAs, mRNAs, and miRNAs of 10 samples in the GSE62589 as described in Section "Materials and Methods." Finally, an osteoporosis-related dysregulated miRNA-mediated lncRNA-mRNA ceRNA network was constructed and was composed of 105 nodes and 515 edges (including eight miRNAs, 24 mRNAs, and 73 lncRNAs) (Figure 2A) (Supplementary Table S2). To further explore the functional implication of the DysCeNet, we performed functional enrichment analysis for mRNAs in the DysCeNet and found that mRNAs in the DysCeNet were significantly enriched in blood vessel development, NIK/NF-kappaB signaling, bone mineralization involved in bone maturation, osteoclast differentiation, and cell aging.

\section{Identification of Potential IncRNA Biomarkers for the Osteoporosis Risk}

To identify potential lncRNA biomarkers for the osteoporosis risk, we performed feature selection for lncRNAs in the DysCeNet using a random forest model. Finally, 25 lncRNAs of 73 lncRNAs in the DysCeNet were identified as potential biomarkers for the osteoporosis risk according to their discriminative power using OOB error (Table 1). Of them, 10 lncRNA biomarkers are up-regulated and 15 lncRNA biomarkers are down-regulated in high-BMD subjects compared
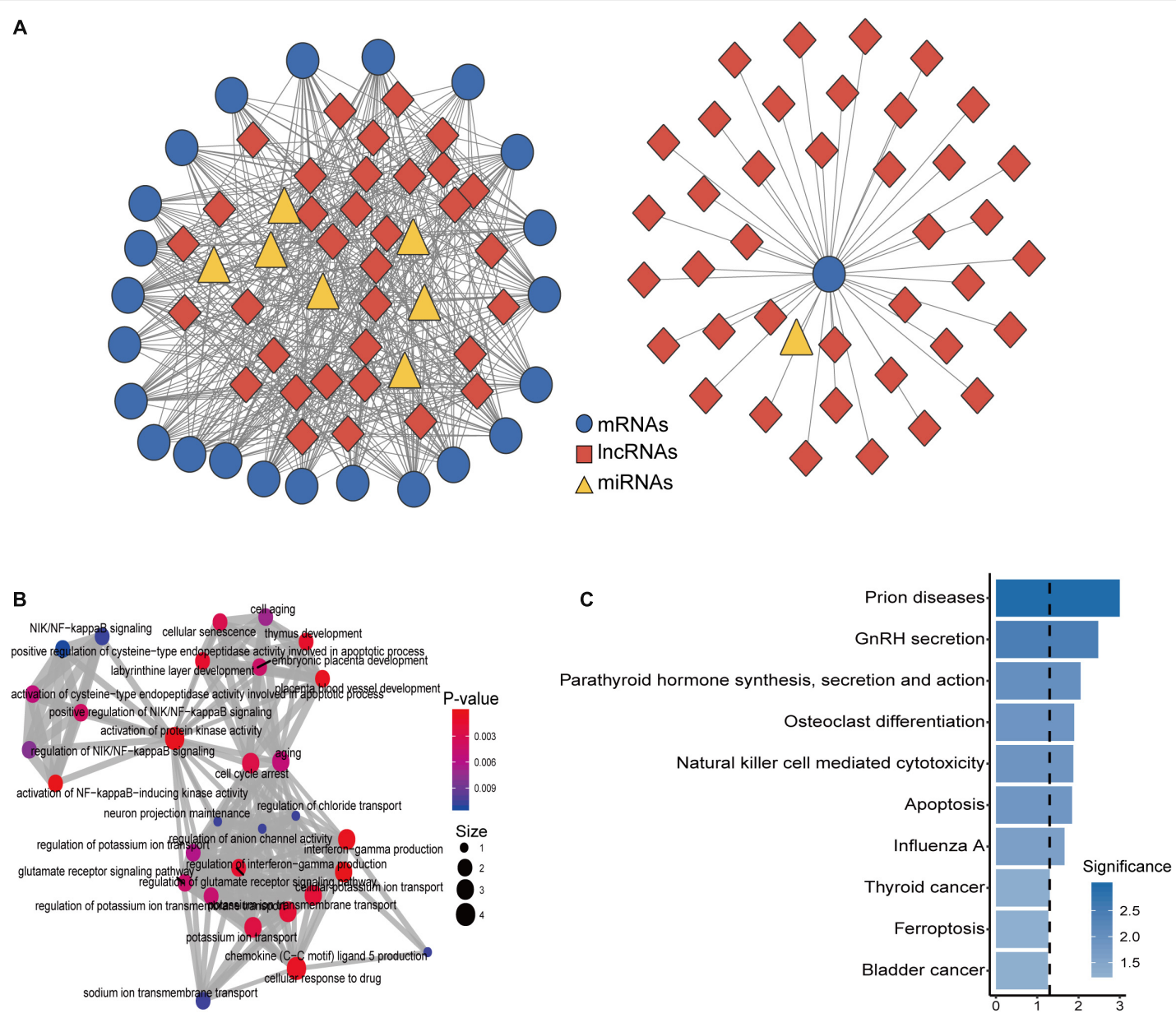

FIGURE 2 | Construction and characterization of dysregulated IncRNA-associated ceRNA network (DysCeNet). (A) A global view of the DysCeNet in osteoporosis. (B) The functional enrichment map of GO terms for mRNAs as ceRNA counterparts of IncRNA biomarkers. (C) Enriched KEGG pathways for mRNAs as ceRNA counterparts of IncRNA biomarkers. 
TABLE 1 | Genomic information of 25 IncRNA biomarkers with ceRNA activity for osteoporosis risk.

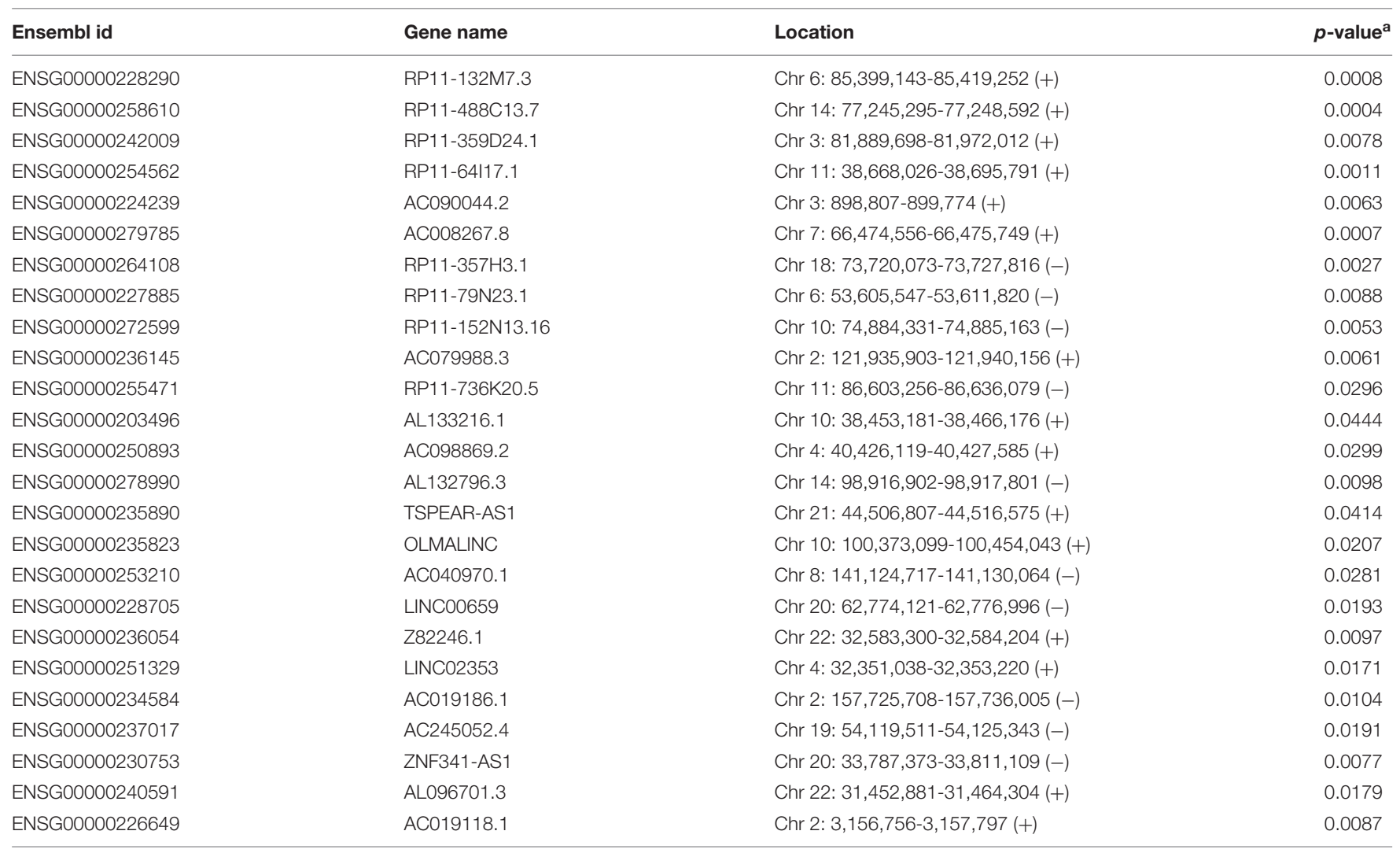

${ }^{a} p$-value was obtained from differential expression analysis.

with low-BMD subjects (Figure 3A). To test whether these 25 lncRNA biomarkers could efficiently distinguish highand low-BMD subjects, we performed hierarchical clustering for 10 samples in the GSE62589 according to the expression pattern of 25 lncRNA biomarkers. As shown in Figure 3B, all 10 samples were classified into two clusters according to the expression pattern of seven lncRNA biomarkers with 100\% accuracy. The results of hierarchical clustering demonstrated the potential of $25 \operatorname{lncRNAs}$ as diagnostic biomarkers for osteoporosis risk. Therefore, a random forest-based classifier composed of 25 lncRNA biomarkers was developed. The performance of the RF-25lncRNA for predicting osteoporosis risk was evaluated in the GSE62589 dataset using the LOOCV procedure, in which nine samples were used as the training set and the remaining one was served as the test sample. Results of performance evaluation showed that the RF25lncRNA achieves a perfect predictive performance in distinguishing high- and low-BMD subjects with an AUC of 1.0 (Figure 3C).

\section{Further Validation of IncRNA Biomarkers for the Osteoporosis Risk in Two Other Independent Datasets}

To test the robustness of the lncRNA biomarkers for the osteoporosis risk, 25 lncRNA biomarkers were applied to the independent GSE56814 dataset. The RF-25lncRNA correctly classified 11 of 15 low-BMD subjects and nine of 16 high-BMD subjects, achieving an AUC of 0.733 (95\% CI: 0.553-0.913) with an accuracy of $64.5 \%$ (Figure $4 \mathrm{~A}$ ).

The performance of the IncRNA biomarkers was further tested in another independent GSE13850 dataset. However, transcriptome gene expression data of the GSE13850 was profiled on the HG-U133A platform, and only two lncRNAs (RP11488C13.7 and RP11-152N13.16) of 25 lncRNA biomarkers in the RF-25lncRNA were covered on the HG-U133A platform. When two lncRNA biomarkers (RP11-488C13.7 and RP11152N13.16) were applied to the 20 samples of the GSE13850, two lncRNA biomarkers (RP11-488C13.7 and RP11-152N13.16) correctly classified seven of 10 low-BMD subjects and seven of 10 high-BMD subjects, achieving an AUC of 0.66 (95\% CI: 0.396-0.924) with an accuracy of 70\% (Figure 4B).

\section{DISCUSSION}

Early detection and intervention for osteoporosis are crucial to prevent fragility fractures and delay disease progression. Traditional markers for osteoporosis risk are BMD, vitamin D, alkaline phosphatase, and so on (Parveen et al., 2019). Increasing evidence has suggested that altered molecular profiles contributed to the osteoporosis and outcome, which provided novel insights into molecular basis of osteoporosis and also highlighted the potential of molecular factors as markers for 
A

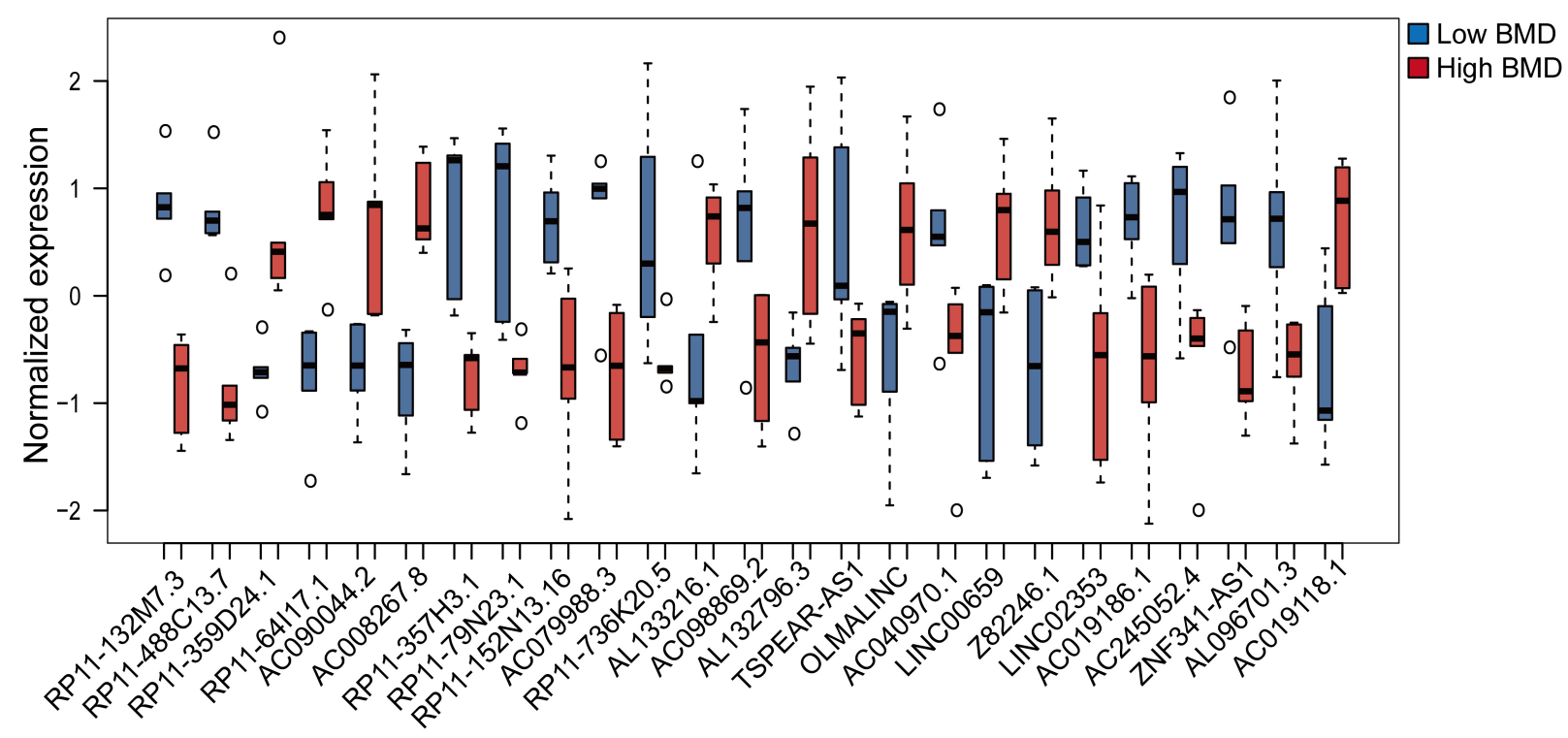

B

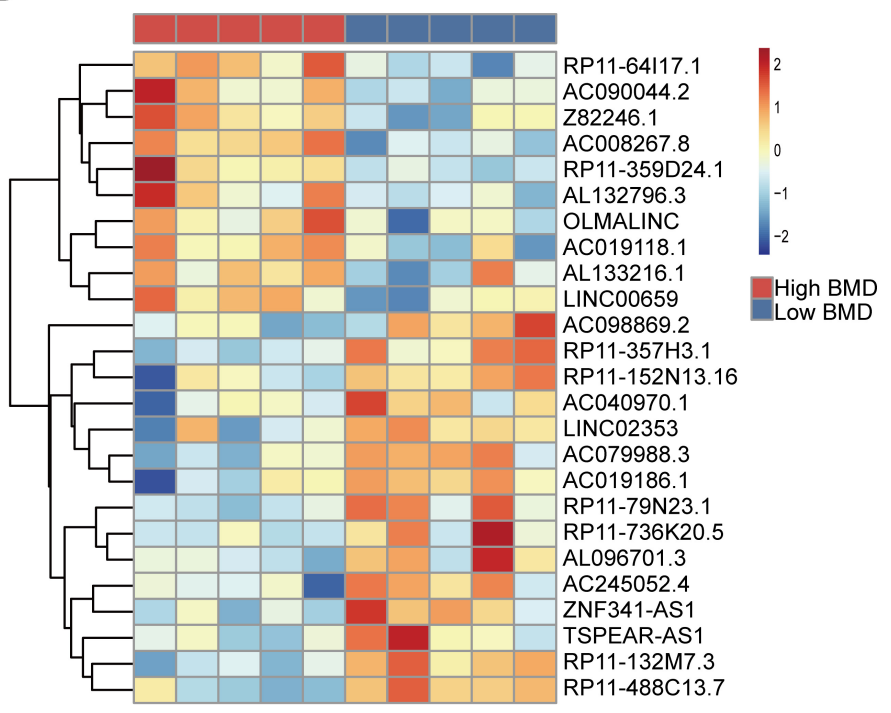

C

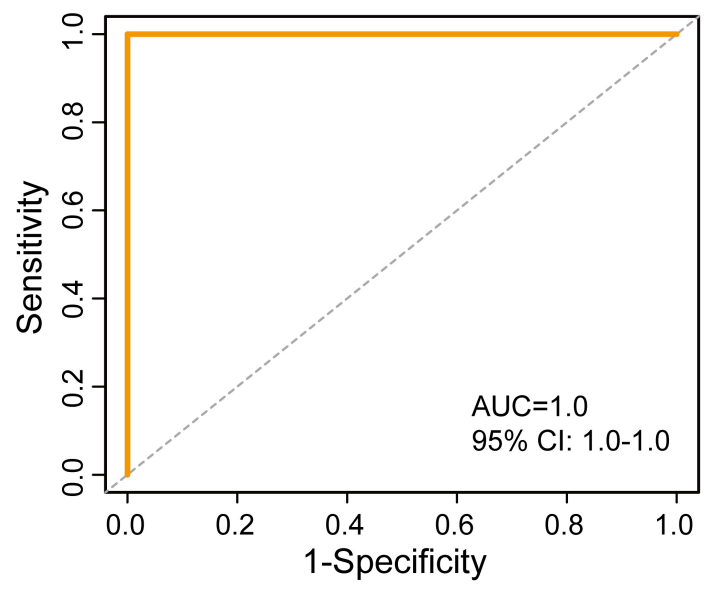

FIGURE 3 | Performance evaluation of identified 25 IncRNA biomarkers in the discovery dataset. (A) Expression pattern of 2525 IncRNA biomarkers in high-BMD and low-BMD subjects. (B) Hierarchical clustering heatmap and dendrogram of 10 samples based on expression patterns of 25 IncRNA biomarkers. (C) The ROC curves of RF-25IncRNA in the GSE62589 dataset.

osteoporosis diagnosis and prognosis (Yu et al., 2013; Makitie et al., 2018; Gong et al., 2019; Ukon et al., 2019). Biomarker identification has been proven to be an effective way to recognize people at high risk for developing osteoporosis and have attracted much attention in the clinical decision-making for osteoporosis management. Previous studies have focused on mRNA profiles and miRNA profiles and identified some candidate mRNA- or miRNA-based biomarkers. For example, circulating miRNAs, $h s a-m i R-122-5 p$ and $h s a-m i R-4516$, have been found to be diagnostic biomarkers for osteoporosis risk (Mandourah et al., 2018). Another study by Seeliger et al. (2014) also identified five freely circulating miRNAs associated with osteoporosis fractures. There is increasing evidence that lncRNAs also play important roles in the pathogenesis of osteoporosis (Peng et al., 2018; Feng et al., 2019; Liu et al., 2019). In this study, we obtained lncRNA profiles of osteoporosis patients by repurposing array probes on publicly available microarray data and performed genome-wide analysis for expression patterns of lncRNAs, miRNAs, and mRNAs between high- and low-BMD subjects. A total of 68 mRNAs, 11 miRNAs, and $95 \operatorname{lncRNAs}$ were found to be associated with osteoporosis, which provided a candidate resource for 
A

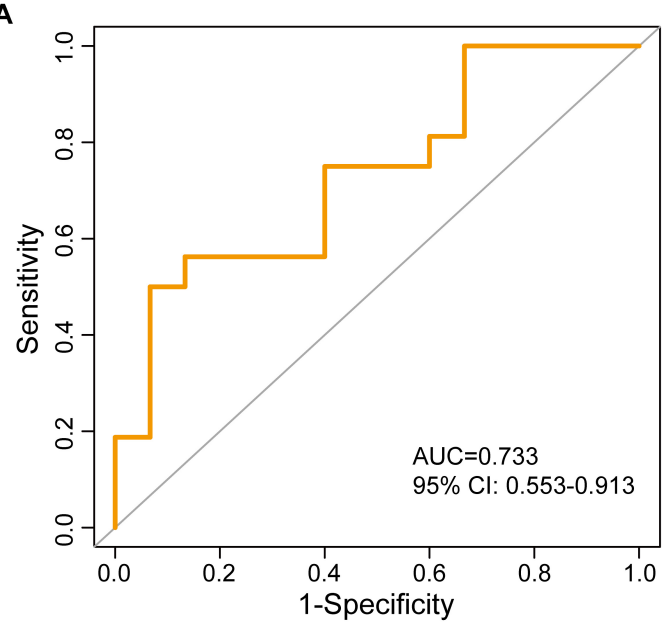

B

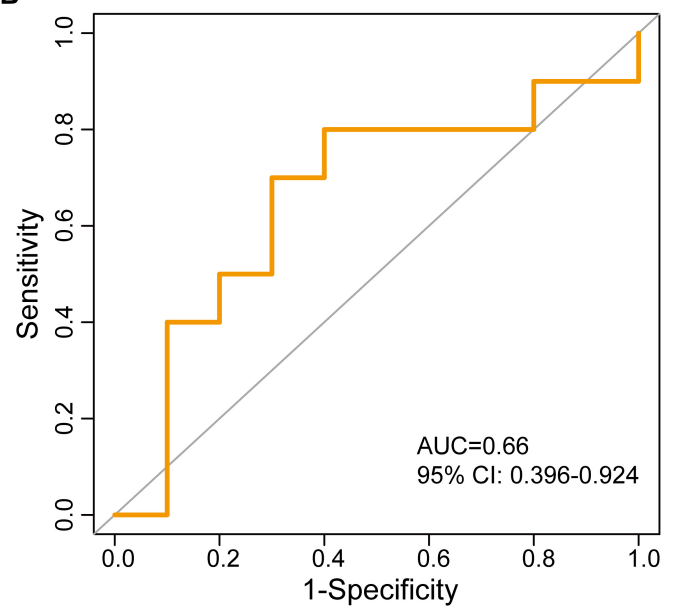

FIGURE 4 | Independent validation of identified 25 IncRNA biomarkers in two independent testing datasets. (A) The ROC curves of RF-25IncRNA in the GSE56814 dataset. (B) The ROC curves of two IncRNA biomarkers (RP11-488C13.7 and RP11-152N13.16) in the GSE13850 dataset.

experimental scientist for studying the molecular mechanism of osteoporosis.

Through many candidate osteoporosis-related mRNAs, miRNAs, and lncRNAs were identified in our study, regulatory relationships and mechanisms among these different types of RNA molecules in the development of osteoporosis are still unknown. It is well known that different RNA molecules can act as ceRNAs to communicate with and co-regulate each other by competing for binding to shared miRNAs (Tay et al., 2014). LncRNAs have been reported as key components of the ceRNA-mediated regulatory network, and aberrant miRNA-mediated lncRNA-mRNA ceRNA crosstalk has been implicated in many human complex diseases, including cancers. However, functional roles and regulatory mechanisms of lncRNA as ceRNAs in the development of osteoporosis and their potential implication for osteoporosis are largely unexplored. To explore the ceRNA activity of lncRNAs in the development of osteoporosis, we performed integrated analysis for paired expression profiles of dysregulated 68 mRNAs, 11 miRNAs, and 95 lncRNAs and miRNA-target regulatory relationship based on "ceRNA hypothesis," and constructed an osteoporosis-related miRNA-mediated dysregulated lncRNA-mRNA ceRNA network (DysCeNet) composed of 105 nodes and 515 edges (including eight miRNAs, 24 mRNAs, and 73 lncRNAs). Network analysis suggested that a large proportion of deregulated lncRNAs $(76.8 \%$, 73/95) in osteoporosis function as ceRNA and communicated with 24 mRNAs by competing for eight common miRNAs (Figure 2A), which implied that extensive variation in miRNAs and lncRNAs disrupted the miRNA-mediated lncRNA-mRNA ceRNA regulatory network contributing to osteoporosis at the post-transcriptional level. Functional analysis through functional enrichment analysis for mRNAs in the DysCeNet found that mRNAs as ceRNA counterparts of lncRNA biomarkers were involved in known osteoporosis or bone metabolism-related biological progression and pathways, including blood vessel development, NIK/NF-kappaB signaling, bone mineralization involved in bone maturation, osteoclast differentiation, and cell aging (Figures 2B,C). For example, blood vessels in the bone play vital roles for the formation of new bone and promoting blood vessel growth could reverse the weakening of bones and treat osteoporosis (Sivaraj and Adams, 2016). NIK/NF-kappaB signaling has been shown to play an important role in the positive and negative regulation of cytokinemediated osteoclast formation and activation (Boyce et al., 2015). These results suggested that dysregulated expression of lncRNA ceRNAs and the resultant perturbation in miRNAmediated IncRNA-mRNA crosstalk in the DysCeNet are involved in osteoporosis-biological processes and contributed to the osteoporosis.

A large number of studies have indicated the superior potential of lncRNAs as diagnostic and prognostic biomarkers compared with protein-coding genes due to the fact that lncRNAs were expressed in much more cell- type-, tissue-, and disease-specific patterns that are closely more associated with their function (Hauptman and Glavaè, 2013; Chen et al., 2018). IncRNA biomarkers have been widely investigated and identified in various cancers during the past years (Zhou et al., 2018a,b, 2019; Bao et al., 2019). To explore the potential application of lncRNAs with ceRNA activity as diagnostic biomarkers for osteoporosis risk, we performed random forest-based feature selection for 73 lncRNAs with ceRNA activity and identified 25 of 73 lncRNAs as potential diagnostic biomarkers. To accelerate the clinical application, we also developed a random forest-based classifier (RF-25lncRNA) composed of seven lncRNA biomarkers and test the performance of the RF-25lncRNA in different osteoporosis datasets. Performance evaluation of the LOOCV procedure showed that the RF-25lncRNA achieved a good performance in distinguishing high- and low-BMD subjects in three osteoporosis datasets. These results demonstrated that 
seven IncRNA with ceRNA activity may become reliable and powerful epigenetic diagnostic biomarkers for early detection of osteoporosis risk.

There are some limitations to this study. First, the performance of newly identified lncRNA biomarkers was validated only in three osteoporosis datasets because of no other publicly available osteoporosis datasets. Second, the biological functions of newly identified lncRNA biomarkers are unknown, although they were found to have ceRNA activity in our study. Therefore, more laboratory and clinical researches were needed.

\section{DATA AVAILABILITY STATEMENT}

Publicly available datasets were analyzed in this study. This data can be found here: Three osteoporosis datasets were obtained from the Gene Expression Omnibus (GEO) database (GSE62589, https://www.ncbi.nlm.nih. gov/geo/query/acc.cgi?acc=GSE62589, GSE56814, https:// www.ncbi.nlm.nih.gov/geo/query/acc.cgi?acc=GSE56814, and

\section{REFERENCES}

Al Anouti, F., Taha, Z., Shamim, S., Khalaf, K., Al Kaabi, L., and Alsafar, H. J. (2019). An insight into the paradigms of osteoporosis: from genetics to biomechanics. Bone Rep. 11:100216. doi: 10.1016/j.bonr.2019.100216

Bao, S., Zhao, H., Yuan, J., Fan, D., Zhang, Z., Su, J., et al. (2019). Computational identification of mutator-derived lncRNA signatures of genome instability for improving the clinical outcome of cancers: a case study in breast cancer. Brief Bioinform. [Epub ahead of print]

Boyce, B. F., Xiu, Y., Li, J., Xing, L., and Yao, Z. J. E. (2015). NF-(B-mediated regulation of osteoclastogenesis. Endocrinol. Metab. 30, 35-44.

Bunch, H. (2018). Gene regulation of mammalian long non-coding RNA. Mol. Genet. Genomics 293, 1-15. doi: 10.1007/s00438-017-1370-9

Burge, R., Dawson-Hughes, B., Solomon, D. H., Wong, J. B., King, A., and Tosteson, A. (2007). Incidence and economic burden of osteoporosis-related fractures in the United States, 2005-2025. J. Bone. Miner. Res. 22, 465-475. doi: 10.1359/jbmr.061113

Chen, L., Zhang, Y.-H., Pan, X., Liu, M., Wang, S., Huang, T., et al. (2018). Tissue expression difference between mRNAs and lncRNAs. Eur. Rev. Med. Pharmacol. Sci. 19:3416. doi: 10.3390/ijms19113416

Chen, X., Yang, L., Ge, D., Wang, W., Yin, Z., Yan, J., et al. (2019). Long noncoding RNA XIST promotes osteoporosis through inhibiting bone marrow mesenchymal stem cell differentiation. Exp. Ther. Med. 17, 803-811. doi: 10. 3892/etm.2018.7033

Durinck, S., Spellman, P. T., Birney, E., and Huber, W. (2009). Mapping identifiers for the integration of genomic datasets with the R/Bioconductor package biomaRt. Nat. Protoc. 4, 1184-1191. doi: 10.1038/nprot.2009.97

Feng, J., Wang, J., and Li, C. H. (2019). LncRNA GAS5 overexpression alleviates the development of osteoporosis through promoting osteogenic differentiation of MSCs via targeting microRNA-498 to regulate RUNX2. Eur. Rev. Med. Pharmacol.Sci. 23, 7757-7765. doi: 10.26355/eurrev_201909_18985

Garnero, P. (2017). The utility of biomarkers in osteoporosis management. Mol. Diagn. Ther. 21, 401-418. doi: 10.1007/s40291-017-0272-1

Gong, R., Ren, S., Chen, M., Wang, Y., Zhang, G., Shi, L., et al. (2019). Bioinformatics analysis reveals the altered gene expression of patients with postmenopausal osteoporosis using liuweidihuang pills treatment. Biomed. Res. Int. 2019:1907906. doi: 10.1155/2019/1907906

Guo, X., Gao, L., Wang, Y., Chiu, D. K., Wang, T., and Deng, Y. (2016). Advances in long non-coding RNAs: identification, structure prediction and function annotation. Brief Funct. Genomics 15, 38-46. doi: 10.1093/bfgp/elv022
GSE13850, https://www.ncbi.nlm.nih.gov/geo/query/acc.cgi? acc $=$ GSE13850).

\section{AUTHOR CONTRIBUTIONS}

YZ conceived and designed the experiments. MZ and LC analyzed the data. MZ wrote the manuscript. All authors read and approved the final manuscript.

\section{SUPPLEMENTARY MATERIAL}

The Supplementary Material for this article can be found online at: https://www.frontiersin.org/articles/10.3389/fcell.2020.00184/ full\#supplementary-material

TABLE S1 | List of differentially expressed miRNAs, mRNAs and IncRNAs between high-BMD subjects and low-BMD subjects.

TABLE S2 | An osteoporosis-related dysregulated miRNA-mediated IncRNA-mRNA ceRNA network.

Hauptman, N., and Glavaè, D. (2013). Long non-coding RNA in cancer. Noncoding RNA Res. 14, 4655-4669.

Huang, R., Wu, J., Zheng, Z., Wang, G., Song, D., Yan, P., et al. (2019a). The construction and analysis of ceRNA network and Patterns of immune infiltration in Mesothelioma with bone metastasis. Front. Bioeng. Biotechnol. 7:257. doi: $10.3389 /$ fbioe. 2019.00257

Huang, R., Zeng, Z., Li, G., Song, D., Yan, P., Yin, H., et al. (2019b). The construction and comprehensive analysis of ceRNA networks and tumorinfiltrating immune cells in bone metastatic melanoma. Front. Genet. 10:828. doi: $10.3389 /$ fgene. 2019.00828

Jiang, H., and Wong, W. H. (2008). SeqMap: mapping massive amount of oligonucleotides to the genome. Bioinformatics 24, 2395-2396. doi: 10.1093/ bioinformatics/btn429

Kornienko, A. E., Guenzl, P. M., Barlow, D. P., and Pauler, F. M. (2013). Gene regulation by the act of long non-coding RNA transcription. BMC Biol. 11:59. doi: 10.1186/1741-7007-11-59

Lai, H.-Y., Zhang, Z.-Y., Su, Z.-D., Su, W., Ding, H., Chen, W., et al. (2019). iProEP: a computational predictor for predicting promoter. Mol. Ther. Nucleic Acids 17, 337-346. doi: 10.1016/j.omtn.2019. 05.028

Li, J. H., Liu, S., Zhou, H., Qu, L. H., and Yang, J. H. (2014). starBase v2.0: decoding miRNA-ceRNA, miRNA-ncRNA and protein-RNA interaction networks from large-scale CLIP-Seq data. Nucleic Acids Res. 42, D92-D97. doi: 10.1093/nar/ gkt1248

Liu, S., Huang, H., Chai, S., Wei, H., Huang, J., and Wan, L. (2019). Expression profile analysis of long non-coding RNA in skeletal muscle of osteoporosis by microarray and bioinformatics. J. Biol. Eng. 13:50. doi: 10.1186/s13036-0190180-5

Lv, H., Zhang, Z.-M., Li, S.-H., Tan, J.-X., Chen, W., and Lin, H. (2019). Evaluation of different computational methods on 5-methylcytosine sites identification. Brief Bioinform. [Epub ahead of print],

Maass, P. G., Luft, F. C., and Bähring, S. (2014). Long non-coding RNA in health and disease. J. Mol. Med. 92, 337-346.

Makitie, R. E., Hackl, M., Niinimaki, R., Kakko, S., Grillari, J., and Makitie, O. (2018). Altered MicroRNA profile in osteoporosis caused by impaired WNT signaling. J. Clin. Endocrinol. Metab. 103, 1985-1996. doi: 10.1210/jc.201702585

Mandourah, A. Y., Ranganath, L., Barraclough, R., Vinjamuri, S., Hof, R. V. T., Hamill, S., et al. (2018). Circulating microRNAs as potential diagnostic biomarkers for osteoporosis. Sci. Rep. 8, 1-10. 
Mei, B., Wang, Y., Ye, W., Huang, H., Zhou, Q., Chen, Y., et al. (2019). LncRNA ZBTB40-IT1 modulated by osteoporosis GWAS risk SNPs suppresses osteogenesis. Biol. Med. 138, 151-166. doi: 10.1007/s00439-019-01969-y

Mercer, T. R., and Mattick, J. S. (2013). Structure and function of long noncoding RNAs in epigenetic regulation. Nat. Struct. Mol. Biol. 20, 300-307. doi: $10.1038 / \mathrm{nsmb} .2480$

Merico, D., Isserlin, R., Stueker, O., Emili, A., and Bader, G. D. (2010). Enrichment map: a network-based method for gene-set enrichment visualization and interpretation. PLoS One 5:e13984. doi: 10.1371/journal.pone.001 3984

Parveen, B., Parveen, A., and Vohora, D. (2019). Biomarkers of osteoporosis: an update. Endocr. Metab. Immune Disord. Drug Targets 19, 895-912. doi: 10.2174/ 1871530319666190204165207

Pelechano, V., and Steinmetz, L. M. (2013). Gene regulation by antisense transcription. Nat. Rev. Genet. 14, 880-893. doi: 10.1038/nrg3594

Peng, S., Cao, L., He, S., Zhong, Y., Ma, H., Zhang, Y., et al. (2018). An overview of long non-coding RNAs involved in bone regeneration from mesenchymal stem cells. Stem Cells Int. 2018:8273648. doi: 10.1155/2018/8273648

Quinn, J. J., and Chang, H. Y. (2016). Unique features of long non-coding RNA biogenesis and function. Nat. Rev. Genet. 17, 47-62. doi: 10.1038/nrg.2015.10

Ritchie, M. E., Phipson, B., Wu, D., Hu, Y., Law, C. W., Shi, W., et al. (2015). limma powers differential expression analyses for RNA-sequencing and microarray studies. Nucleic Acids Res. 43:e47. doi: 10.1093/nar/gkv007

Seeliger, C., Karpinski, K., Haug, A. T., Vester, H., Schmitt, A., Bauer, J. S., et al. (2014). Five freely circulating miRNAs and bone tissue miRNAs are associated with osteoporotic fractures. J. Bone Miner. Res. 29, 1718-1728. doi: 10.1002/ jbmr. 2175

Silva, A. M., Moura, S. R., Teixeira, J. H., Barbosa, M. A., Santos, S. G., and Almeida, M. I. (2019). Long non-coding RNAs: a missing link in osteoporosis. Bone Res. 7:10. doi: 10.1038/s41413-019-0048-9

Sivaraj, K. K., and Adams, R. H. (2016). Blood vessel formation and function in bone. Development 143, 2706-2715. doi: 10.1242/dev.136861

Sun, J., Zhang, Z., Bao, S., Yan, C., Hou, P., Wu, N., et al. (2020). Identification of tumor immune infiltration-associated lncRNAs for improving prognosis and immunotherapy response of patients with non-small cell lung cancer. J. Immunother. Cancer 8:e00110. doi: 10.1136/jitc-2019-000110

Tan, J.-X., Li, S.-H., Zhang, Z.-M., Chen, C.-X., Chen, W., Tang, H., et al. (2019). Identification of hormone binding proteins based on machine learning methods. Math. Biosci. Eng. 16, 2466-2480. doi: 10.3934/mbe.2019123

Tay, Y., Rinn, J., and Pandolfi, P. P. (2014). The multilayered complexity of ceRNA crosstalk and competition. Nature 505, 344-352. doi: 10.1038/nature12986
Toth, R., Schiffmann, H., Hube-Magg, C., Buscheck, F., Hoflmayer, D., Weidemann, S., et al. (2019). Random forest-based modelling to detect biomarkers for prostate cancer progression. Clin. Epigenetics 11:148. doi: 10. 1186/s13148-019-0736-8

Tu, K. N., Lie, J. D., Wan, C. K. V., Cameron, M., Austel, A. G., Nguyen, J. K., et al. (2018). Osteoporosis: a review of treatment options. PT 43, 92-104.

Ukon, Y., Makino, T., Kodama, J., Tsukazaki, H., Tateiwa, D., Yoshikawa, H., et al. (2019). Molecular-based treatment strategies for osteoporosis: a literature review. Int. J. Mol. Sci. 20:E2557. doi: 10.3390/ijms20102557

Yu, G., Wang, L., Li, Y., Ma, Z., and Li, Y. (2013). Identification of drug candidate for osteoporosis by computational bioinformatics analysis of gene expression profile. Eur. J. Med. Res. 18:5. doi: 10.1186/2047-783X-18-5

Yu, G., Wang, L. G., Han, Y., and He, Q. Y. (2012). clusterProfiler: an R package for comparing biological themes among gene clusters. OMICS 16, 284-287. doi: 10.1089/omi.2011.0118

Zhou, M., Diao, Z., Yue, X., Chen, Y., Zhao, H., Cheng, L., et al. (2016). Construction and analysis of dysregulated lncRNA-associated ceRNA network identified novel lncRNA biomarkers for early diagnosis of human pancreatic cancer. Oncotarget 7, 56383-56394. doi: 10.18632/oncotarget.10891

Zhou, M., Hu, L., Zhang, Z., Wu, N., Sun, J., and Su, J. (2018a). Recurrenceassociated long non-coding RNA signature for determining the risk of recurrence in patients with colon cancer. Mol. Ther. Nucleic Acids 12, 518-529. doi: 10.1016/j.omtn.2018.06.007

Zhou, M., Zhang, Z., Zhao, H., Bao, S., Cheng, L., and Sun, J. (2018b). An immunerelated Six-lncRNA signature to improve prognosis prediction of glioblastoma multiforme. Mol. Neurobiol. 55, 3684-3697. doi: 10.1007/s12035-017-0572-9

Zhou, M., Zhao, H., Wang, X., Sun, J., and Su, J. (2019). Analysis of long non-coding RNAs highlights region-specific altered expression patterns and diagnostic roles in Alzheimer's disease. Brief Bioinform. 20, 598-608. doi: 10. 1093/bib/bby021

Conflict of Interest: The authors declare that the research was conducted in the absence of any commercial or financial relationships that could be construed as a potential conflict of interest.

Copyright $(2020$ Zhang, Cheng and Zhang. This is an open-access article distributed under the terms of the Creative Commons Attribution License (CC BY). The use, distribution or reproduction in other forums is permitted, provided the original author(s) and the copyright owner(s) are credited and that the original publication in this journal is cited, in accordance with accepted academic practice. No use, distribution or reproduction is permitted which does not comply with these terms. 